\title{
VARIA
}

\section{La crónica deportiva como recurso de escritura en ELE/L2. Un enfoque procesual}

\section{The Match Report as a writing tool in the teaching of Spanish as a Foreign Language / Second Language. A processual approach}

\author{
Francisco Núñez-Román \\ Department of Teaching of Spanish and Literature and Integrated Philologies, \\ Universidad de Sevilla, Facultad de Ciencias de la Educación, c/ Pirotecnia sn, Sevilla, Spain \\ fnroman@us.es \\ Emilio J. Gallardo-Saborido \\ Escuela de Estudios Hispano-Americanos, CSIC, c/ Alfonso XII, 16. 41002, Seville, Spain ${ }^{1}$ \\ emilio.gallardo@csic.es
}

\begin{abstract}
This paper stands up for the defence of writing as a hugely valuable resource for teaching Spanish as a Foreign Language or a Second Language. Specifically, it pursues two general targets: a) to highlight the importance of considering writing into the classroom of Spanish as a Foreign Language as a process that has to be constantly coached and developed; b) to emphasise how this way of teaching provides pedagogical benefits that go further the development of the writing ability itself, since it has an impact on other students' general cognitive features. Methodologically, this text begins with a literature review that theoretically underpins the proposed targets, and, subsequently, our didactic proposal is exemplified by the use of a specific type of text (the match report). Then, a practical teaching resource is obtained as final result, which is supported by the recent literature on this issue. Finally, this contribution aspires to be implemented in different educative contexts by teachers of Spanish as a Foreign Language.
\end{abstract}

Keywords: second foreign language, Spanish language, written expression, match report, processual approach

\footnotetext{
${ }^{1}$ La escritura de este artículo tuvo lugar siendo este autor profesor de la Universidad de Sevilla.
} 


\section{EL USO DE LA ESCRITURA EN EL AULA DE ELE}

La expresión escrita suele ocupar un espacio secundario en las clases de lengua extranjera, en las que se privilegian los enfoques comunicativos basados en las destrezas orales, consideradas más útiles para la vida cotidiana del aprendiz (Buyse, 2010). Sin embargo, vivimos en una sociedad letrada, que experimenta una revitalización del código escrito frente a la oralidad. Actualmente, se escribe de manera más habitual, en diferentes soportes (sobre todo en medios electrónicos, como WhatsApp, Facebook, correo electrónico, etc.) y con finalidades diversas (dar una opinión, compartir un estado de ánimo, hacer una petición, etc.). No obstante, la presencia de la escritura no se refleja en las clases de lengua extranjera en la misma medida en la que está presente en nuestras sociedades letradas.

Como docentes de lenguas extranjeras, y en concreto de ELE, nos debemos replantear algunas cuestiones claves que ya lanzó Cassany (2005):

1. ¿Qué concepción de la escritura tengo como docente?

2. ¿Qué enseño a escribir en mis clases?

3. ¿Dónde pongo el acento en las tareas de escritura de mis alumnos?

Las respuestas a estas cuestiones determinan no solo nuestra formación como escritores sino también nuestra perspectiva como docentes. De esa manera, la primera pregunta nos indicará si consideramos la escritura como un elemento secundario en el proceso de enseñanza-aprendizaje (actividad de refuerzo de las destrezas orales en los métodos audio-linguales), como un medio de comunicación (práctica con textos reales en los enfoques nocional-funcionales) o como un medio de aprendizaje de otros contenidos (enfoque basado en contenidos). Raramente considera-remos las tareas escritas en la clase de L2 como una oportunidad para aprender los procesos de escritura y los procedimientos y estrategias que se llevan a cabo en la elaboración de un texto escrito (enfoque procesual).

La segunda pregunta, por su parte, nos llevará a enumerar una larga lista de textos escritos que quedan perfectamente recogidos en los documentos de referencia de enseñanza de lenguas extranjeras (como el Marco Común Europeo de Referencia, en adelante MCER, y su desarrollo en el Plan curricular del Instituto Cervantes). Se trata, en definitiva, de una amplia muestra de tipologías textuales, ligadas a las distintas actividades de lengua, con un enfoque orientado a la acción.

Por último, si analizamos qué consideramos importante en los escritos de nuestros alumnos, se harán evidentes las razones por las que les pedimos que escriban: las faltas de ortografía y la sintaxis (escribir para aprender lengua), la macroestructura (los tipos de texto), los contenidos (los elementos culturales), la adecuación (los aspectos socioculturales), etc.

Parece evidente que, habitualmente, no ponemos el acento en el proceso que nuestro alumno inicia cuando le encomendamos la tarea, y esperamos de él, únicamente, un producto final que se ajuste a unas pautas de evaluación. De hecho, como señala Sánchez Castilla (2016) en su estudio sobre las dificultades de escritura de los 
estudiantes en L2, estos manifiestan una mayor preocupación por cuestiones de índole gramatical que por cuestiones procedimentales. Entendemos, erróneamente, que la escritura es una actividad solitaria, silenciosa, en general aburrida y que requiere de un tiempo y un esfuerzo que no podemos dedicar en las horas de clase. Sin embargo, no somos conscientes de que poniendo a disposición del alumno el conocimiento de su propio proceso de escritura, estamos potenciando sus capacidades y desarrollando, a la vez, su competencia lingüística. Sánchez hace notar una de las grandes paradojas de la escritura en L2 al afirmar que "se escribe mucho en una lengua extranjera, tanto dentro como fuera de la clase, pero [...] se enseña a escribir poco" $(2009$, p. 22$)$.

\section{LOS PROCESOS DE ESCRITURA EN L1 Y L2}

Aunque han sido muchos los autores que han elaborado sus propios modelos explicativos sobre el proceso escritor, uno de los modelos que más éxito ha tenido ha sido el propuesto por Flower y Hayes (1981). Esta propuesta, que se enmarca dentro de los modelos cognitivos, se articula en torno a tres elementos: la memoria a largo plazo, la situación comunicativa y los procesos de escritura (Fig. 1).

\section{MODELO DE EXPRESIÓN ESCRITA}

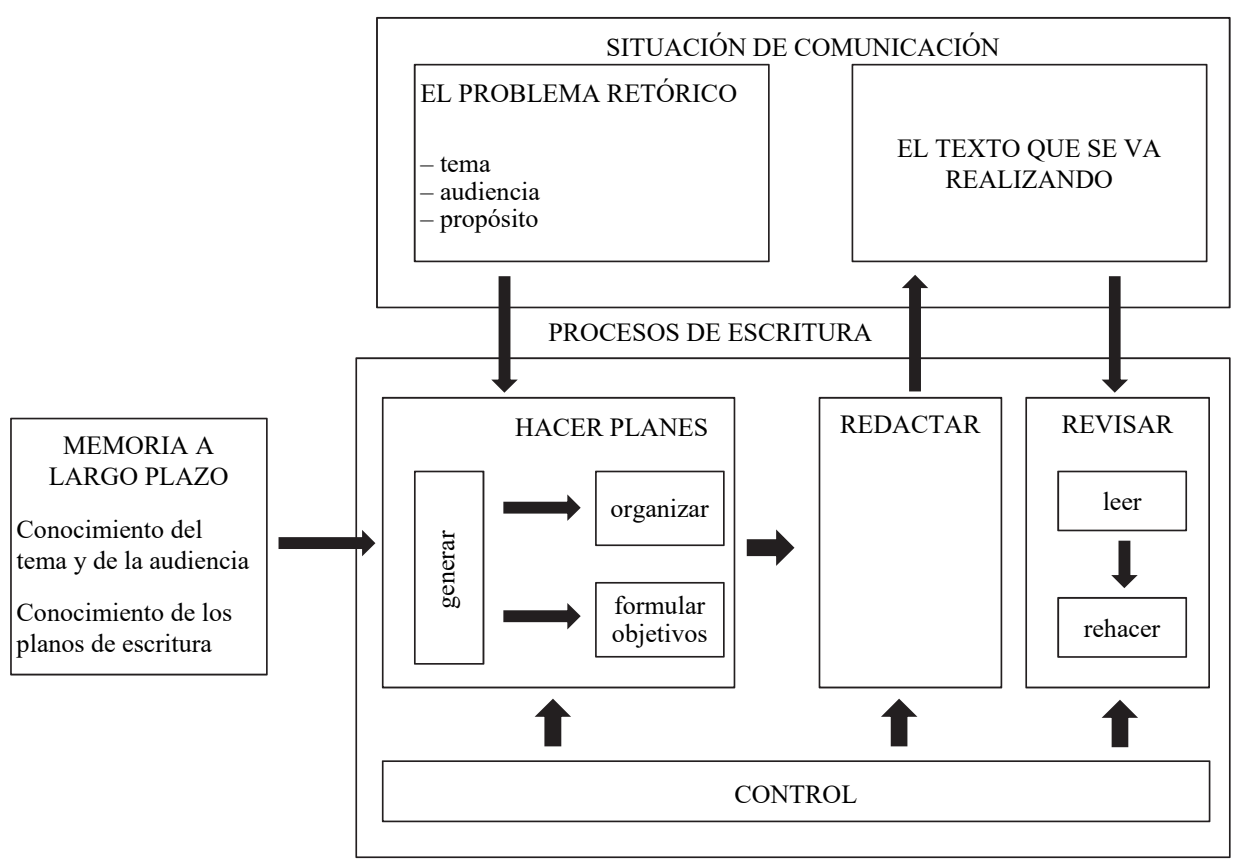

Fig. 1. Modelo de expresión escrita de Flower y Hayes (Cassany, Luna, Sanz, 1994, p. 264, basado en Flower y Hayes, 1981) 
La situación comunicativa hace que el escritor se plantee una serie de cuestiones iniciales, denominadas "problema retórico": sobre qué voy a escribir, a quién va dirigido, qué pretendo conseguir con mi texto. Estos elementos determinan los procesos de escritura, esto es, planificar el texto (generar y organizar las ideas del texto, y formular los objetivos del escrito), redactar o textualizar (traducir en palabras, frases y párrafos los conceptos que conformarán el texto) y revisar (leer, evaluar y rehacer el texto conforme al plan inicial). Estos procesos de escritura no son lineales, sino recursivos, lo que quiere decir que planificamos, redactamos y revisamos constantemente, en el intento de conseguir el escrito más cercano al texto ideal que nos habíamos imaginado al inicio de la tarea. Para que puedan ponerse en marcha estos procedimientos, son necesarios dos elementos: en primer lugar, la memoria a largo plazo, que activa nuestras ideas previas sobre el tema y el conocimiento de la audiencia y el género textual; $y$, en segundo lugar, el control o monitor, que se encarga de gestionar todos los elementos que entran en juego y autorregular el proceso globalmente, de manera que el escritor sea consciente en cada momento de los requerimientos puntuales y las estrategias adecuadas para alcanzar los objetivos de escritura planteados.

Este modelo se centra en "els processos mentals d'elaboració i d'organització de la informació en funció dels temes de què es tracta i dels contextos retòrics en què es produeixen els textos" (Guasch, 2001, p. 12) y no en el conocimiento del código lingüístico. Como resalta Guasch (2001), estos procesos son universales a cualquier lengua, por lo que son el punto de partida ideal para afrontar la enseñanza de la escritura en L1 o en L2.

Esta universalidad, además, se refleja en la relación entre los procesos de composición y la enseñanza de lenguas extranjeras. López González (2013) pone de manifiesto esta similitud entre los procesos de escritura de Flower y Hayes y las estrategias de comunicación que recoge el Marco Común Europeo de Referencia (Tab. 1).

Tab. 1. Procesos de composición y estrategias de comunicación (elaboración propia, adaptado de López González, 2013, p. 73)

\begin{tabular}{|l|l|}
\hline \multicolumn{1}{|c|}{ Proceso de composición } & \multicolumn{1}{c|}{ Estrategias de comunicación } \\
\hline Planificar & Planificación \\
\hline Textualizar & Ejecución \\
\hline \multirow{2}{*}{ Revisar } & Control \\
\cline { 2 - 2 } & Reparación \\
\hline
\end{tabular}

\section{CARACTERÍSTICAS DE LA ESCRITURA EN ELE}

En muchas ocasiones, pensamos que nuestros alumnos son competentes en la escritura en L1, y que esa competencia se puede transferir fácilmente al nuevo contexto de aprendizaje en L2. Sin embargo, afirmaciones de este tipo contienen dos 
tipos de errores: en primer lugar, nuestros alumnos pueden no ser competentes en escritura en L1, debido a las propias características de aprendizaje de esta habilidad (es una práctica situada que requiere un periodo de aprendizaje prolongado en el tiempo); en segundo lugar, no se ha demostrado que se dé una transferencia de habilidades automática, pues escritores expertos en L1 pueden no demostrar la misma competencia escrita en L2, debido, entre otras cuestiones, a la diferente tradición discursiva o a déficits lingüísticos (Guasch, 2001).

Las características de la escritura en L2, por lo tanto, vendrán determinadas por los siguientes aspectos:

a. La competencia escrita del aprendiz;

b. la transferencia de habilidades de escritura de la L1 a la L2;

c. y el dominio de la L2.

Estos factores interactúan entre sí, de manera que las carencias mostradas en alguno de ellos deben ser suplidas por medio de un mayor dominio de los otros factores.

\subsection{La competencia escrita del aprendiz}

La competencia escrita es uno de los conocimientos más difíciles de aprender incluso en lengua materna, ya que esta habilidad supone un aprendizaje prolongado y sobre todo situado (Barton, Hamilton, Ivanič, 2000), es decir, dependiente del contexto social de la escritura. En éste resulta determinante la comunidad discursiva en la que escribimos (laboral, académica, personal, etc.). Cada comunidad discursiva posee sus propios textos con sus propias convenciones, y es necesario un largo proceso de aculturación en el seno de estas comunidades para apropiarse de sus formas de expresión escrita. La culminación de este proceso no suele alcanzarse de manera completa o satisfactoria por el usuario estándar de la lengua en todos los contextos posibles de uso. Por lo tanto, la falta de competencia escrita en L2 refleja principalmente la falta de competencia escrita general del aprendiz, independientemente del nivel de dominio de la L2 que este posea.

Diversos estudios corroboran esta afirmación (Guasch, 2001). En este sentido, se ha podido comprobar que los procesos de composición de escritores expertos en L2 son similares a los procesos de composición de los escritores expertos en L1, tanto en estudios entre sujetos diferentes (nativos vs. no nativos) como en estudios en sujetos individuales (composición en L1 y en L2 del mismo estudiante). Lo mismo sucede con las habilidades escritas de los escritores novatos en ambas lenguas, los cuales tienden a cometer errores similares. Entre las conductas que llevan a cabo los escritores expertos, Guasch (2001, p. 39) destaca las siguientes (Tab. 2). 
Tab. 2. Conductas de escritores expertos en L1 y L2 (elaboración propia a partir de Guasch, 2001, p. 39)

\begin{tabular}{|l|}
\hline \multicolumn{1}{|c|}{ Conductas } \\
\hline Usan estrategias particulares para resolver problemas mientras escriben. \\
\hline Transforman su conocimiento mientras escriben. \\
\hline Usan representaciones mentales complejas para guiar sus decisiones. \\
\hline Producen más contenido efectivo y organizado del discurso en sus composiciones. \\
\hline Interrelacionan los procesos de planificación y producción de forma mucho más elaborada y compleja. \\
\hline Ponen atención a la selección de palabras. \\
\hline
\end{tabular}

Además, diversos estudios han concluido que las habilidades de escritura en L1 son más decisivas en aquellos casos de bajo dominio de la L2, ya que las destrezas adquiridas en L1 son una herramienta facilitadora del proceso escritor en L2 (Hyland, 2003; Silva, 1993). De hecho, el grado de dominio de las habilidades de escritura es un elemento diferenciador de las estrategias que emprenden los aprendices para resolver problemas, siendo más ricas cuanto más control del proceso escritor se tenga (Guasch, 2001).

A pesar de estos datos y aunque los procesos de escritura en L1 y L2 puedan ser similares, hay que resaltar que se dan acciones específicas que diferencian la escritura en L1 y L2. Guasch (2001) enumera algunas de las características de los procesos de escritura en L2 (Tab. 3).

Finalmente, la falta de conocimiento de las convenciones textuales de la L2 puede provocar errores de tipo pragmático: escribir en L2 supone también aprender las convenciones textuales en esa lengua, que pueden (o no) diferir de las de la L1.

Tab. 3. Características de los escritores en L2 (Guasch, 2001, p. 47)

\begin{tabular}{|l|l|}
\hline & \multicolumn{1}{|c|}{ Características de los escritores en L2 } \\
\hline Efectos del menor conocimiento & $\begin{array}{l}\text { No se automatiza el uso de determinados aspectos de la composición. } \\
\text { Conocimientos lingüísticos reducidos. }\end{array}$ \\
\hline Planificación & $\begin{array}{l}\text { Menos atención a los objetivos globales. } \\
\text { Menos planificación global del texto. }\end{array}$ \\
\hline Textualización & $\begin{array}{l}\text { Más pausas que en la composición en lengua materna. } \\
\text { Más consultas de materiales auxiliares. } \\
\text { Atención prioritaria a dar forma lingística a las ideas. } \\
\text { Poca atención a la corrección formal. }\end{array}$ \\
\hline Revisión & $\begin{array}{l}\text { Menor tendencia a producir nuevos borradores. } \\
\text { Más dificultad para atender simultáneamente a los aspectos locales } \\
\text { y globales del texto. }\end{array}$ \\
\hline Proceso de composición en \\
general & $\begin{array}{l}\text { Más implicación en las tareas de composición. } \\
\text { Necesidad de más tiempo para escribir. } \\
\text { Uso de la lengua materna durante la composición. } \\
\text { Descubrimiento simultáneo de ideas y material lingüístico. } \\
\text { Producción de textos más breves. }\end{array}$ \\
\hline
\end{tabular}




\subsection{La transferencia de habilidades de escritura}

Puesto que los procesos de escritura que se llevan a cabo en L1 y L2 son similares, parece plausible proponer la existencia de una transferencia de habilidades de una lengua a otra. Este aspecto, no obstante, se ha mostrado como uno de los puntos más controvertidos sobre la escritura en L2. Mientras que algunos autores han propuesto la transferencia automática de estas habilidades (Cummins, Swain, 1986), diversos estudios han rebatido, en mayor o menor medida, esta hipótesis. En primer lugar, se ha defendido que se produce una transferencia parcial de estas habilidades (Bialystok, 1994). Las nuevas tareas de escritura provocan en el escritor la reorganización de sus conocimientos previos de escritura; esta reorganización estaría causada por las exigencias de la nueva situación y las características del nuevo código (Guasch, 2001). De esta manera, las habilidades no se transferirían de manera directa, sino que se modificarían a causa de la nueva situación de escritura y obligarían a la reestructuración de los conocimientos del escritor. En este sentido, los estudios que muestran cómo individuos con un alto dominio de escritura en L1 presentan carencias en la producción escrita en L2 parecen corroborar esta hipótesis. En segundo lugar, Freedle (1985) defiende que no es posible transferir en absoluto las habilidades de escritura de la L1 a la L2 debido a los contextos específicos en los que se producen los textos, por lo que únicamente es posible transferir microhabilidades individuales ligadas a situaciones de uso muy específicas. Estas microhabilidades, por otro lado, se seleccionarían de entre las denominadas habilidades básicas de escritura en L1, y que posee toda persona letrada en prácticas escritoras fundamentales (la "abstract language performance" de Jones, Tetroe, 1987).

En definitiva, parece que existen determinadas limitaciones, de tipo lingüístico o pragmático, que impiden que los aprendices trasladen con total garantía sus prácticas escritoras de una lengua a otra de manera automática. Aunque se den este tipo de transferencias, estas parecen no ser ni automáticas ni invariables, ya que existen factores que las favorecen o entorpecen. Consideremos, por ejemplo, el dominio de la L2, la reorganización de los conocimientos de escritura del aprendiz (algo que facilita el procesamiento del nuevo material lingüístico) o la toma de conciencia de los procesos de escritura (Guasch, 2001).

\subsection{El dominio de la L2 y el recurso a la L1}

En cualquier proceso de aprendizaje de una L2, el nivel de dominio del nuevo código se convierte en un elemento obstaculizador o facilitador del éxito del proceso. En el caso de la competencia escrita, el conocimiento de las habilidades de escritura en L1 se muestra como un factor más determinante que el dominio de la L2 para la elaboración de buenos textos en esta segunda lengua. En esta línea, Guasch 
(2001) confirma que los escritores expertos en L1 son capaces de atender de manera simultánea a varios aspectos de la composición, siendo más conscientes del carácter complejo de la escritura independientemente de su nivel de L2. Sin embargo, los escritores de nivel intermedio o novatos son mejores o peores según su dominio de la L2. Por lo tanto, el dominio de la L2 adquiere un papel secundario, ya que puede ser un elemento facilitador o entorpecedor de la puesta en marcha de las habilidades de escritura, pero no afecta al grado de dominio de estas habilidades (Guasch, 2001).

Por otro lado, es habitual recurrir a la L1 durante el proceso de composición, sobre todo para superar los bloqueos provocados por la falta de competencia lingüística en L2. Se trata, en definitiva, de aquellos procesos que o bien están determinados por elementos alejados del mundo cultural de la L1 o bien suponen la generación y selección de ideas almacenadas en la memoria a largo plazo del escritor. Sin embargo, algunos autores han señalado los obstáculos que presenta el recurso habitual a la L1 (Moragne e Silva, 1989):

a. Problemas de fluidez en la escritura: al producir un primer borrador en L1, este debe ser traducido a la L2, provocando interrupciones en la composición a causa de los posibles problemas de traducción encontrados.

b. Falta de automatización: procedimientos como la revisión, que deben realizarse de manera sistemática y global, quedan relegados a simples revisiones superficiales al final de la edición del texto.

c. Problemas de tratamiento intercultural: algunos temas de la L2 pueden ser tratados desde la perspectiva de la L1, provocando problemas en la estructuración de los contenidos, que se presentan en formatos propios de la L1 y alejados del mundo cultural de la L2.

Por lo tanto, el recurso a la L1 debe ser una herramienta puntual, que le permita al aprendiz desbloquearse en momentos críticos en los que sea necesario manipular información nueva, pero que no impida el desarrollo de las competencias escritoras en L2 desde una perspectiva interlingüística.

\section{LA ENSEÑANZA DE LA ESCRITURA EN L2 POR MEDIO DE LA ENSEÑANZA DE LOS PROCESOS DE ESCRITURA}

Ni el MCER ni el Plan curricular del Cervantes se posicionan desde el punto de vista metodológico, limitándose a remarcar, en el caso del MCER, que "el enfoque de la metodología del aprendizaje y de la enseñanza tiene que ser integrador, presentando todas las opciones de forma explícita y transparente y evitando las preferencias o el dogmatismo" (Consejo de Europa, 2002, cap. 6.4). Por su parte, el Plan curricular del Instituto Cervantes "concibe los procedimientos de aprendizaje desde la perspectiva de su uso estratégico o condicionado al tipo de tarea al que el aprendiente se enfrente en cada momento" (Instituto Cervantes, 2006, cap. 13), re- 
saltando la necesidad de analizar previamente las exigencias de la tarea para alcanzar un aprendizaje y uso eficaz de la lengua. Alerta, además, de que, si no se usan los procedimientos de manera consciente y adecuada, "podría incurrirse en un uso mecánico de los procedimientos de aprendizaje que no redundaría en el desarrollo de estrategias" (Instituto Cervantes, 2006, cap. 13).

Por lo tanto, sin ánimo de realizar una propuesta dogmática sobre la enseñanza de la escritura en L2, consideramos que nuestra contribución pretende proporcionar a los alumnos, por medio del conocimiento del proceso de escritura, destrezas y habilidades que mejoren sus capacidades cognitivas generales, y por lo tanto, redunden en la mejora de las destreza escrita del sujeto, tanto en L1 como en L2. Además, el enfoque basado en el proceso favorece la autorregulación de la escritura, haciendo al aprendiz más consciente de los procedimientos, las técnicas y la globalidad del proceso.

Desde este punto de vista, hay que resaltar los siguientes aspectos antes de abordar la enseñanza de la escritura de manera procesual (Raimes, 1985, 1987, citado en Guasch, 2001, pp. 40-42):

1. Es necesario adaptar o cambiar las estrategias de escritura en L2, ya que, aunque los procesos de escritura en L1 y L2 puedan ser similares, estas pueden no ser adecuadas a la nueva situación comunicativa. En lugar de atender a las estrategias generales de escritura, es preferible centrarse en estrategias específicas para cada tarea de escritura concreta: generar ideas, organizar ideas, elaborar un párrafo, revisar la temática, etc.

2. Puesto que la competencia escrita no está directamente relacionada con el conocimiento de la L2, es necesario dar a conocer las convenciones de los productos escritos de la lengua meta, por medio de la exposición y el análisis de modelos textuales y de las convenciones sociolingüísticas subyacentes a cada modelo.

\section{TÉCNICAS Y ESTRATEGIAS PARA FAVORECER LA ESCRITURA EN ELE}

Nuestra propuesta se basa en los siguientes ejes:

\subsection{Aprendizaje cooperativo}

La práctica escrita no debe entenderse como una tarea individual: escribir cooperativamente es más productivo y didácticamente más efectivo (Kagan, Kagan, 2009). El trabajo en grupo permite compartir, discutir, conocer las formas de trabajo del compañero, en un proceso de reflexión del propio proceso de escritura, en el que podemos observar nuestras debilidades y fortalezas, y activar estrategias de mejora. 
Para ello, propondremos la combinación de tareas individuales y tareas grupales, dependiendo de los requisitos de cada una. Los momentos de planificación y revisión son más rentables si se trabajan en grupo, mientras que la textualización de parte o de la totalidad del escrito puede realizarse de manera individual (Kruse, 2013).

Es importante, además, que los alumnos entiendan la filosofía del trabajo colaborativo: no se trata, en última instancia, de sumar productos individuales, sino de crear un producto común fruto de la reflexión y del crecimiento intelectual de todos los participantes.

\subsection{Reflexión sobre la propia escritura}

Hablar sobre el proceso de escritura permite exteriorizar y reflexionar sobre los procedimientos, las actitudes y las dificultades que el escritor se encuentra durante el proceso de composición. Sirve para evidenciar lo que habitualmente es inconsciente, $\mathrm{y}$, sobre todo, ayuda al aprendiz a secuenciar las tareas que ha realizado y darse cuenta de las que le quedan por hacer.

La reflexión sobre la propia escritura puede hacerse de manera individual en los niveles más altos (en forma de diario de escritura) o bien de manera colectiva en los niveles más bajos (por medio de debates en el seno de cada equipo de trabajo). Esta reflexión en voz alta, si se hace en L2, aumenta además el input lingüístico al que está sometido el aprendiz, al tiempo que favorece la interacción con otros actores (coautor, lector, coevaluador) y consolida el proceso de escritura.

\subsection{Conocimiento del proceso}

La escritura en segunda lengua es una de las tareas que más ansiedad provoca a los estudiantes. Según se desprende en Sánchez Castilla (2016), las cuestiones gramaticales, el escaso tiempo disponible para elaborar el texto y la evaluación son los principales factores ansiógenos. La concepción de la escritura como un recorrido amplio reduce el estrés de las tareas inmediatas (evitando las redacciones que se ordenan de un día a otro a final de la clase) y permite consolidar los conocimientos adquiridos. El alumno puede percibir las diferentes fases del proceso, lo que le proporciona seguridad al saber en cada momento en qué punto del recorrido se encuentra (qué ha hecho y qué debe hacer). Por otro lado, permite al aprendiz centrar sus esfuerzos en la tarea actual, sin preocuparse por tareas futuras o pasadas, eliminando la ansiedad creada ante el volumen de trabajo.

Además, al concebir la escritura como un proceso, y no como un producto, se le otorga una mayor importancia a los borradores. Estos productos intermedios, habitualmente desechados y menospreciados, suponen la auténtica piedra de toque del 
proceso de aprendizaje, pues permiten afrontar las dificultades y trabajar en su mejora mientras el aprendiz tiene tiempo de consolidar sus conocimientos (Albarrán Santiago, 2005). Los borradores son, en definitiva, herramientas de aprendizaje altamente productivas y rentables.

Finalmente, si nos centramos en el proceso de escritura, eliminamos la relación entre error y penalización que en ocasiones bloquea a los estudiantes. Aunque es bien sabido que el nivel de compromiso con la tarea de escritores en L2 es mayor que el de los escritores en L1, al tiempo que estos muestran menos miedo a cometer errores, ya que entienden que se encuentran en un proceso de aprendizaje (Guasch, 1995), debemos transmitir que la tarea de escritura es una actividad placentera y útil, y convertirla en una oportunidad para que el alumno investigue, aprenda y se exprese sobre aquello que le interesa.

\section{UNA PROPUESTA PRÁCTICA: "UNA LIGA PARA CONTARLA"}

Para concluir ofrecemos una propuesta didáctica para realizar en el aula de ELE, en concreto en el nivel B2. Basada en un enfoque de escritura procesual toma como punto de partida un posible centro de interés compartido y un espacio de encuentro intercultural como son los deportes. Asimismo, se sustenta en el conocimiento y creación de un tipo textual específico: la crónica deportiva. Por último, aprovecha las técnicas y estrategias para favorecer la escritura en ELE que especificábamos en el apartado anterior. Esto es:

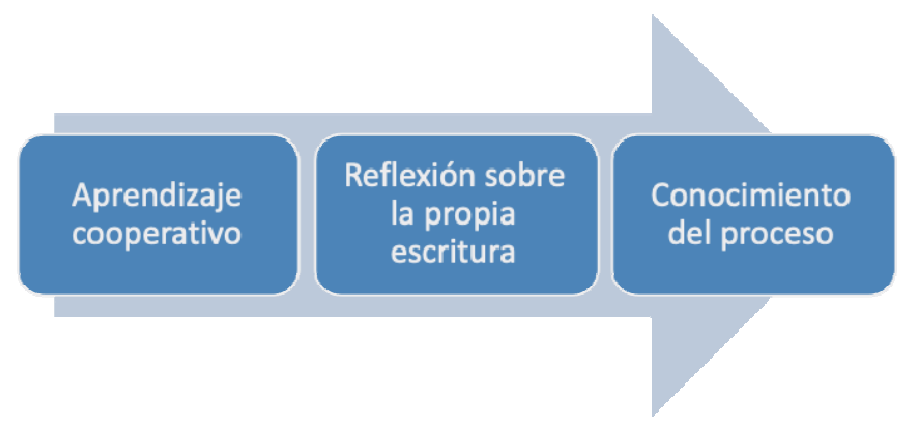

Fig. 2. Técnicas y estrategias para favorecer la escritura en ELE

Aunque la propuesta puede resultar lo suficientemente flexible como para ser adaptada a distintas secuencias de enseñanza, entendemos que una posible rutina de aprendizaje se podría pautar para una semana de trabajo, realizado por parejas, y conducente a la producción de una crónica deportiva por equipo, de modo que la 
suma de todas las del grupo-clase componga el resumen de lo ocurrido en la jornada de la Liga que hayamos seleccionado. En cuanto a la secuenciación del proceso de trabajo, aprovecharemos algunas de las indicaciones que Díaz y Aymerich (2003) ofrecen para producir textos a partir del enfoque procesual. Resumimos todo el proceso a continuación.

En la primera etapa de escritura que tendremos en consideración ("primeros pasos en la planificación") $)^{2}$, los alumnos aúnan sus conocimientos lingüísticos y del mundo con aspectos como el propósito, el lector y el tipo de texto (Díaz, Aymerich, 2003, p. 96). En ella, podemos recurrir a estrategias como los asociaciogramas, los esquemas previos, la documentación y la lluvia de ideas (Díaz, Aymerich, 2003, p. 97). En el caso de la crónica deportiva, partiremos del análisis de los conocimientos previos, tanto lingüísticos como extralingüísticos, que sobre el periodismo deportivo posean nuestros alumnos. A ello, le sumaremos una reflexión sobre las características de la crónica deportiva como tipo textual en la que incluiremos la cuestión del propósito y del potencial lector. Por último -aunque no necesariamente en este orden-, les ofreceremos ejemplos de textos reales a partir de los cuales podamos trabajar. En este caso, hemos seleccionado el artículo "La Undécima más dramática" (Rubén Jiménez, Marca, 28 de mayo de 2016).

En la segunda etapa de escritura que aquí consideraremos ("primeras formulaciones"), se produce "la transición de la planificación a la configuración de lo planificado, plasmado en una primera formulación coherente" (Díaz, Aymerich, 2003, p. 97). Siguiendo con nuestro ejemplo, los estudiantes pueden proceder a realizar las siguientes tareas:

a. Seleccionar el partido que deberán narrar en sus crónicas.

b. Analizar el o los modelos de partida, identificando usos lingüísticos propios del texto meta. Así, en la crónica que estamos usando de ejemplo podrían proceder a familiarizarse con:

i. Léxico o colocaciones particulares del ámbito deportivo ("los tacos", "balón colgado").

ii. Estructuras sintácticas propias del periodismo deportivo como la enumeración climática: "El gol de Ramos, el gol de la final, el gol de la Décima, el gol de la Undécima".

iii. Otros recursos literarios (metáforas, metonimias, sinécdoques) que implican el conocimiento de referentes y alusiones específicas del ámbito futbolístico español y que tejen una red de significados compartidos entre

\footnotetext{
${ }^{2}$ En el epígrafe "Un modelo del proceso de enseñanza de la escritura", Díaz y Aymerich (2003, pp. 93-104) mencionan los siguientes subapartados: ejercicios de escritura; primeros pasos en la planificación del texto; el trabajo con material de consulta; primeras formulaciones; el mapa lineal de ideas o el análisis de la estructura; formulación lineal; bosquejo y borrador; la revisión. En nuestro caso, no mencionaremos explícitamente ni el epígrafe inicial sobre ejercicios de escritura, ni el dedicado al trabajo con material de consulta.
} 
el autor y el lector: "el vecino del once", "la historia negra del Manzanares", "la nación blanca".

c. Organización de las ideas iniciales que incluirán en la crónica, proyectando así un primer esbozo de una posible estructura textual.

Seguidamente pasaremos a la etapa denominada "mapa lineal de ideas o análisis de la escritura", útil para propiciar una reflexión detenida sobre "cómo podría ser la estructura del texto a partir de asociaciones de ideas" (Díaz, Aymerich, 2003, p. 98). Para ello recurriríamos al análisis de la estructura de la crónica deportiva a partir de varios modelos reales. Díaz \& Aymerich animan a estimular el trabajo de los alumnos con preguntas que les ayuden a organizar sus mapas lineales y jerárquicos. Se trataría de cuestiones del tipo: “¿Qué viene primero? ¿Por qué?”; “¿Cuáles son los puntos de mayor peso?”; o, por ejemplo, “¿Qué tiene que ir junto y qué no?” (p. 98). Para nuestros intereses particulares, una vez transcurrido el partido del que haremos la crónica, procederemos a confeccionar un borrador de la estructura del texto teniendo en cuenta elementos esenciales en la narración de un partido de fútbol como las alineaciones, el desarrollo del primer y del segundo tiempo, y el resultado final.

En la etapa próxima ("formulación lineal") incidiremos en cómo se deben tejer las relaciones entre las ideas que hemos ido desarrollando para la confección de nuestro texto y los fragmentos de los que en este punto podemos disponer. Díaz y Aymerich recuerdan que: "Hasta aquí nuestro texto está solamente planificado", y algo más adelante: "El estudiante debe aprender una serie de estrategias y medios lingüísticos para poder tejer un texto a partir de frases y plasmar su idea inicial" (2003, p. 99). En concreto, hacen referencia a los conectores y a las relaciones de coreferencia. En este sentido, los alumnos pueden haber practicado previamente estas cuestiones, pero también podemos proponerles buscar y analizar funcionalmente los conectores y las relaciones de co-referencia en el texto o textos que estemos usando como modelo. Asimismo, pueden disponer como material de consulta de tablas donde se recojan los distintos conectores agrupados por categorías funcionales (véase, por ejemplo, Cassany, 2011, pp. 155-157).

Llegados a la fase de bosquejo y borrador, “(...) tenemos que alentar a los estudiantes a tachar, a comenzar de nuevo, a reformular, probar, cambiar, perfilar. La 'formulación lineal' significa lanzar un primer bosquejo, hacer un borrador que posteriormente podamos pasar a limpio" (Díaz y Aymerich, 2003, p. 99). Así pues, debemos concienciar a los estudiantes de la naturaleza recursiva del proceso de escritura, animándolos a no quedar satisfechos por la primera versión obtenida. En ese propósito nos puede ayudar abordar el proceso de reescritura trabajando con distintos tipos de correcciones, de modo que nos posibiliten revisar desde las capas microtextuales hasta las supertextuales, superando así la tradicional preponderancia de las correcciones de índole ortográfica.

Por último, la etapa final de "revisión", como apuntan Díaz y Aymerich (2003, p. 101), puede potenciarse invitando al alumno a ponerse en el papel del lector 
o solicitando la ayuda de un coautor. La revisión de las crónicas que hayan producido nuestros estudiantes puede dar lugar, en primera instancia, a una reflexión por parejas en torno a las expectativas del equipo y los objetivos docentes perseguidos, para, a continuación, proceder a una dinámica de intercambio de textos que involucre a todo el grupo-clase. Ésta se puede concretar en lecturas cruzadas de las diversas crónicas entre los distintos equipos de trabajo, al tiempo que se les ofrecen a los autores nuevas visiones y revisiones sobre los textos.

La secuencia didáctica presentada podría crecer en interés para el alumnado si en ella se introdujera alguna dinámica de gamification o ludificación. En este sentido, se podrían hacer emparejamientos de los distintos equipos e ir decidiendo cuáles pasan cada ronda clasificatoria, de modo que colectivamente se consensúe quiénes han producido el mejor texto y, por lo tanto, se conviertan en los campeones de nuestra particular liga de escritura. De esta manera, añadiríamos no sólo un componente lúdico, sino que estimularíamos la reflexión sobre nuestras capacidades como escritores, abriendo un nuevo espacio en clase para el diálogo sobre este asunto.

\section{CONCLUSIONES}

La enseñanza de la escritura en ELE basada en el enfoque procesual permite mejorar la competencia escrita del aprendiz no solo en L2, sino también en su lengua materna, dado el carácter universal de los procesos cognitivos de escritura y la posibilidad de transferencia de conocimientos de una lengua a otra. Para que la secuencia de aprendizaje alcance sus objetivos, es necesario adaptar las estrategias de escritura al contexto de la L2, lo que obliga a abordar estrategias específicas centradas en las tareas que componen cada fase del proceso. De este modo, se favorece el conocimiento de la globalidad del proceso y se asegura la adquisición de procedimientos y técnicas que permiten autorregular la propia escritura.

En el diseño de secuencias didácticas basadas en el enfoque procesual hay que tener en cuenta tanto la competencia escrita en L1 del aprendiz, que puede presentar importantes déficits, la posibilidad de transferir esos conocimientos de una lengua a otra, con las limitaciones mencionadas anteriormente, y el nivel de dominio de la L2, que puede suponer un importante obstáculo para la puesta en marcha del proceso de enseñanza-aprendizaje. Por ello, es importante dar a conocer las convenciones textuales oportunas y formar en diversas técnicas de escritura, además de propiciar la reflexión explícita sobre la misma. De este modo, ayudaremos a nuestros alumnos a perfeccionar su competencia escrita, en particular, pero además fortaleceremos su competencia lingüística general.

En concreto, nuestra propuesta tiene en cuenta estos aspectos y combina elementos que invitan a la reflexión sobre el propio proceso de escritura (la concreción del destinatario, la tipología y finalidad del texto o el desarrollo del mapa lineal de 
ideas) y las particularidades de la escritura en L2 (análisis sintáctico y léxicosemántico de la L2 y comparación de modelos textuales). Del mismo modo, la selección de la temática (la crónica deportiva) permite poner en contacto elementos interculturales presentes en el día a día del alumnado y resaltar las metáforas y expresiones propias del deporte que proliferan en el uso cotidiano de la lengua.

$\mathrm{Si}$ a todo lo anterior sumamos las bondades educativas que aporta el aprendizaje cooperativo, el aprovechamiento de los centros de interés de los estudiantes y las posibilidades que abre la ludificación de las actividades de aula, estaremos mucho más cerca de lograr el principal objetivo planteado: escribir con eficiencia y de forma placentera en L2. 


\section{BIBLIOGRAFÍA}

Albarrán Santiago, M. (2005). La evaluación en el enfoque procesual de la composición escrita. Educere, 9(31), 545-552.

Barton, D., Hamilton, M., Ivanič, R. (2000). Situated literacies: reading and writing in context. London/New York: Routledge.

Bialystok, E. (1994). Analysis and control in the development of second language proficiency. Studies in Second Language Acquisition, 16, 157-168.

Buyse, K. (2010). La expresión escrita en la clase de ELE: ingredientes esenciales, sazonados o no con TIC. Mosaico. Revista para la Promoción y Apoyo a la Enseñanza del Español, 26, 4-12.

Cassany, D. (2005). Expresión escrita en L2/ELE. Madrid: Arco Libros.

Cassany, D. (2011). La cocina de la escritura. Barcelona: Anagrama.

Cassany, D., Luna, M., Sanz, G. (1994). Enseñar lengua. Barcelona: Graó.

Consejo de Europa (2002). Marco común europeo de referencia para las lenguas: aprendizaje, enseñanza, evaluación. Madrid: Ministerio de Educación, Cultura y Deporte.

Cummins, J., Swain, M. (1986). Bilingualism in education: aspects of theory, research, and practice. Londres: Longman.

Díaz, L., Aymerich, M. (2003). La destreza escrita. Madrid: Edelsa.

Flower, L., Hayes, J. (1981). A cognitive process of writing. College Composition and Comunication, 32 (4), 365-387.

Freedle, R.O. (1985). Achieving Cognitive Synthesis of Separate Language Skills: Implication for Improving. En C.N. Hedley, A.N. Baratta (eds.), Contexts of Reading (pp. 107-126). Norwood, NJ: Ablex.

Guasch, O. (1995). Els processos d'escriptura en segones llengües. Articles: Revista de didàctica la llengua i de la literatura, 5, 13-22.

Guasch, O. (2001). L'escriptura en segones llengües. Barcelona: Graó.

Hyland, K. (2003). Second language writing. New York: Cambridge University Press.

Instituto Cervantes (2006). Plan Curricular del Instituto Cervantes. Niveles de Referencia para el Español. Madrid: Biblioteca Nueva.

Jiménez, R. (28.05.2016). La Undécima más dramática. Marca. Recuperado de: http://www. marca.com/eventos/marcador/futbol/2015_16/champions/final/rma_atm/.

Jones, C., Tetroe, J. (1987). Composing in a second language. In A. Matsuhashi (ed.), Writing in Real Time: Modelling production processes (pp. 34-57). Norwood, NJ: Ablex.

Kagan, S., Kagan, M. (2009). Kagan Cooperative Learning. San Clemente, CA: Kagan Publishing, cop.

Kruse, O. (2013). Perspective on Academic Writing in European Higher Education: Genres, Practices, and Competences. Revista de Docencia Universitaria, 11(1), 37-58.

López González, A. (2013). Aprender y enseñar a escribir en español lengua extranjera. In M. Spychała, L.S. Bustinza, J. Hadaś (eds), El alumno de ELE: un alumno extraordinario. Marco teórico y propuestas prácticas para trabajar en la clase de Español Lengua Extranjera (ELE) (pp. 67-84). Poznan: Comité Central de las Olimpiadas de Español Asociación Neofilológica Polaca.

Moragne e Silva, M. (1989). A Study of Composing in a First and a Second Language. Texas Papers in Foreign Language Education, 1 (2), 132-151.

Sánchez, D. (2009). La expresión escrita en clase de ELE. II Jornadas de formación de profesores de ELE: estrategias de enseñanza y aprendizaje del español en China. Suplementos MarcoEle, 8, 1-41. Recuperado de: http://marcoele.com/descargas/china/sanchez_expresionescrita.pdf. 
Sánchez Castilla, F.M. (2016). Influencia de la ansiedad sobre la producción escrita en español como lengua extranjera. MarcoEle. Revista de didáctica ELE, 22, 1-22. Recuperado de: http://marcoele.com/descargas/22/sanchez-ansiedad_escritura_.pdf.

Silva, T. (1993). Toward an understanding of the distinct nature of L2 writing: The ESL research and its implications. TESOL quarterly, 27(4), 657-677. 